\title{
Rate of failure of indirect decompression in lateral single-position surgery: clinical results
}

\author{
J. Alex Thomas, MD, ${ }^{1}$ Christopher I. M. Thomason, BS, ${ }^{1}$ Brett A. Braly, MD, ${ }^{2}$ and \\ Cristiano M. Menezes, MD, $\mathrm{PhD}^{3}$
}

${ }^{1}$ Atlantic Neurosurgical and Spine Specialists, Wilmington, North Carolina; ${ }^{2}$ The Spine Clinic of Oklahoma City, Oklahoma City, Oklahoma; and ${ }^{3} \mathrm{HVC}$ Hospital and Columna Institute, Belo Horizonte, Brazil

OBJECTIVE Lateral single-position surgery (LSPS) of the lumbar spine generally involves anterior lumbar interbody fusion (ALIF) performed in the lateral position (LALIF) at L5-S1 with or without lateral lumbar interbody fusion (LLIF) at L4-5 and above, followed by bilateral pedicle screw fixation (PSF) without repositioning the patient. One obstacle to more widespread adoption of LSPS is the perceived need for direct decompression of the neural elements, which typically requires flipping the patient to the prone position. The purpose of this study was to examine the rate of failure of indirect decompression in a cohort of patients undergoing LSPS from L4 to S1.

METHODS A multicenter, post hoc analysis was undertaken from prospectively collected data of patients at 3 institutions who underwent LALIF at L5-S1 with or without LLIF at L4-5 with bilateral PSF in the lateral decubitus position between March 2018 and March 2020. Inclusion criteria were symptoms of radiculopathy or neurogenic claudication, central or foraminal stenosis (regardless of degree or etiology), and indication for interbody fusion at L5-S1 or L4-S1. Patients with back pain only; those who were younger than 18 years; those with tumor, trauma, or suspicion of infection; those needing revision surgery; and patients who required greater than 2 levels of fusion were excluded. Baseline patient demographic information and surgical data were collected and analyzed. The number of patients in whom indirect decompression failed was recorded and each individual case of failure was analyzed.

RESULTS A total of 178 consecutive patients underwent LSPS during the time period (105 patients underwent LALIF at L5-S1 and 73 patients underwent LALIF at L5-S1 with LLIF at L4-5). The mean follow-up duration was $10.9 \pm 6.5$ months. Bilateral PSF was placed with the patient in the lateral decubitus position in 149 patients, and there were 29 stand-alone cases. The mean case time was $101.9 \pm 41.5$ minutes: 79.3 minutes for single-level cases and 134.5 minutes for 2-level cases. Three patients (1.7\%) required reoperation for failure of indirect decompression.

CONCLUSIONS The rate of failure of indirect decompression in LSPS from L4 to S1 is exceedingly low. This low risk of failure should be weighed against the risks associated with direct decompression as well as the risks of the extra operative time needed to perform this decompression.

https://thejns.org/doi/abs/10.3171/2020.6.FOCUS20375

KEYWORDS LLIF; lateral lumbar interbody fusion; ALIF; anterior lumbar interbody fusion; indirect decompression; lateral single-position surgery

$\mathrm{L}$ ATERAL lumbar interbody fusion (LLIF) is a minimally invasive variation of anterior lumbar interbody fusion (ALIF) that allows for powerful indirect decompression by increasing the disc height, foraminal height, and central canal diameter. ${ }^{1-5}$ One limitation of LLIF, however, is the lack of a solution to address pathology at L5-S1. Recently, we have contributed to the development of a stepwise surgical technique to perform ALIF in the lateral decubitus position (LALIF). LALIF allows for the anterior placement of large intervertebral spacers via a minimally invasive corridor with the patient remaining in the lateral decubitus position. ${ }^{6} \mathrm{Cou}-$ pling LALIF at L5-S1 and LLIF at L4-5 and above with the placement of bilateral pedicle screw fixation (PSF)

ABBREVIATIONS ALIF = anterior lumbar interbody fusion; $\mathrm{DDD}=$ degenerative disc disease; $\mathrm{EBL}=$ estimated blood loss; LALIF = lateral ALIF; LLIF = lateral lumbar interbody fusion; LSPS = lateral single-position surgery; PSF = pedicle screw fixation.

SUBMITTED May 1, 2020. ACCEPTED June 1, 2020.

INCLUDE WHEN CITING DOI: 10.3171/2020.6.FOCUS20375. 
in the lateral decubitus position allows for a strategy of lateral single-position surgery (LSPS) for robust $360^{\circ} \mathrm{fu}-$ sion without the need for patient repositioning..$^{711}$

One potential obstacle to the adoption of LSPS is the assumed need for direct decompression of the neural elements. Most commonly, a direct decompression requires repositioning the patient prone, thus negating the efficiency benefits of LSPS. ${ }^{2,12,13}$ A complete commitment to indirect decompression in LALIF and LLIF would allow maximal benefits of LSPS to be obtained. The purpose of this study was to examine the multicenter adequacy of indirect decompression when performing LSPS from L4 to $\mathrm{S} 1$.

\section{Methods \\ Study Design and Patient Population}

A multicenter post hoc analysis was undertaken from prospectively collected data of consecutive patients who underwent LALIF at L5-S1 with or without LLIF at L4-5 between March 2018 and March 2020 at 3 institutions. Patients who required supplemental fixation underwent placement of bilateral PSF in the lateral decubitus position. The New Hanover Medical Center (Wilmington, NC) institutional review board approved this study.

Patients were included if they had symptoms of radiculopathy or neurogenic claudication and indication for interbody fusion at L5-S1 or L4-S1. Patients with back pain only; those who were younger than 18 years; those with tumor, trauma, or suspicion of infection; or those needing revision surgery were excluded. Patients who required greater than 2 levels of fusion were also excluded. Patients with stenosis were not excluded regardless of the severity of stenosis and regardless of the etiology of stenosis (e.g., free herniated disc fragment, facet cyst, or bony stenosis). Thus, all patients who underwent interbody fusion at the L5-S1 or L4-S1 levels during this 2-year time period were committed to the mechanism of action of indirect decompression. Cases were performed by 3 different spine surgeons; 2 of these surgeons (J.A.T. and B.A.B.) worked with approach surgeons at L5-S1, while 1 surgeon (C.M.M.) performed his own approaches.

Patient demographics as well as surgical data including estimated blood loss (EBL), operating room time, length of stay, and perioperative complications were collected and analyzed. Individual cases of failure of indirect decompression were also analyzed.

\section{Surgical Technique}

The patient is placed on a standard operating room table in a lateral decubitus position, with either the right side or left side up (if L4-5 is to be included in the fusion construct, a left-side-up approach is preferred). The back of the patient should be situated about 3 inches from the edge of the bed padding to facilitate placement of downside PSF. The C-arm should be positioned such that it enters the field from the front of the patient at an angle to not interfere with the surgeon's access to the abdomen (Fig. 1). The patient is secured to the table with tape crossing the iliac crest obliquely, to properly secure the pelvis but to not interfere with access to a low-lying incision. We then

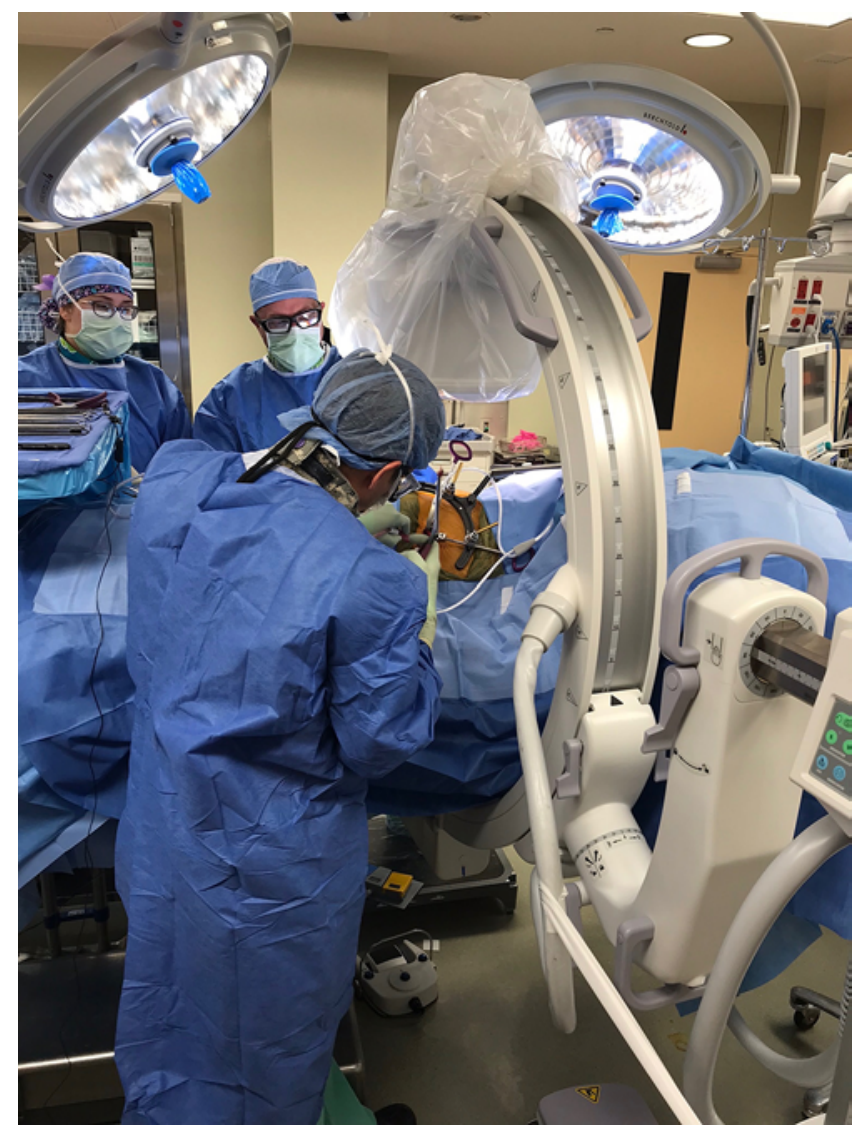

FIG. 1. Photograph of the surgeon working on the abdominal side of the patient during LALIF at L5-S1 (patient is right side up with feet at the left side of the image). Note that the C-arm is kept on the abdominal side of the patient so that it does not interfere with the transition to LLIF or percutaneous PSF. The $\mathrm{C}$-arm enters at an angle, and the large cable is taped back to maximize the surgeon's working area.

use a K-wire to mark the angle of the disc space on the lateral aspect of the iliac crest. This mark not only helps plan the incision but also is useful for the approach surgeon to use for reorientation during the approach if necessary. A 3- to 4-cm transverse incision is planned centered over a point just medial to the midpoint between the anterior spine of the iliac crest and the lateral border of the rectus sheath. It is important that the medial pole of the incision extends just over the lateral border of the rectus sheath to facilitate access into the retroperitoneum.

After the incision is made, the rectus fascia is incised to enter the preperitoneal space. There, the surgeon identifies the plane that separates the lateral border of the rectus from the overlying oblique and transversalis. Subsequently, the surgeon works through the retroperitoneal fat in a trajectory "up and over" the peritoneum following the body wall. Eventually, the psoas muscle and anterior aspect of the spine can be palpated. The surgeon continues to work medial to the vessels with gentle finger dissection sweeping any remaining peritoneum downward and away from the working area. Gentle finger dissection and/ or Kittner dissectors are used to clear adventitious tissue and the hypogastric plexus away from the anterior disc 


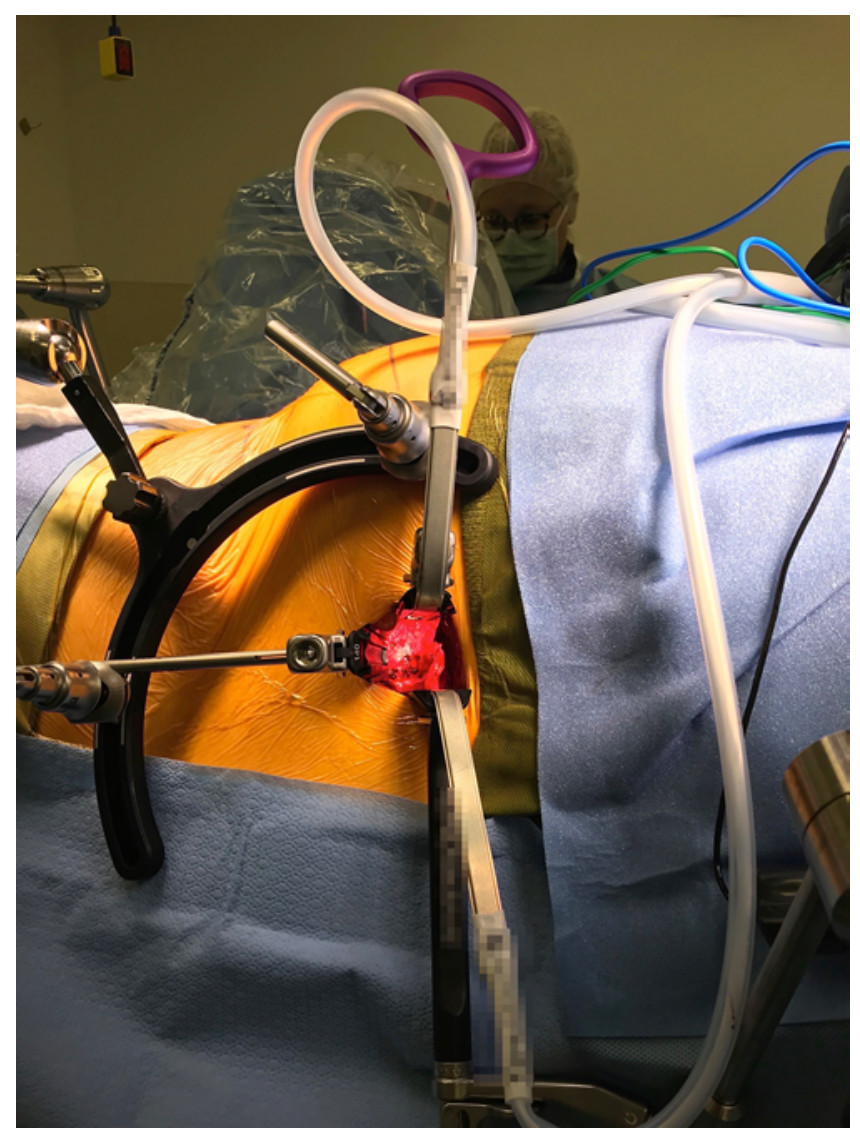

FIG. 2. Photograph showing the LALIF retractor in place over the L5-S1 disc space. The patient is left side up, and the head is at the left side of the image. The lateral (up) blade retracts left iliac vessels, the medial (down) blade retracts right iliac vessels, and the cranial blade protects bifurcation.

annulus. Here, the advantage of the lateral decubitus position will become obvious, as gravity will gently retract the peritoneum away from the working area. Lastly, the middle sacral vein is identified and coagulated to allow for mobilization of the left iliac vein. Retractor blades are inserted and sequentially attached to the carbon fiber frame of the bed-mounted retractor system (Fig. 2). Disc preparation, graft trialing, and insertion proceed via a direct midline approach as with a traditional supine ALIF.

Once LALIF at L5-S1 is complete, the spine surgeon can transition to the LLIF or PSF portion of the procedure, the techniques for which have been described previously. ${ }^{7,14}$ These portions can be started while the anterior incision is closed (Fig. 3).

\section{Results}

A total of 178 patients were included in the study: 105 patients underwent LALIF at L5-S1, and 73 patients underwent LALIF at L5-S1 with LLIF at L4-5. The mean follow-up was $10.9 \pm 6.5$ months. There were 91 males and 87 females. The mean BMI was $29.6 \pm 5.2 \mathrm{~kg} / \mathrm{m}^{2}$. Indications for surgery included degenerative disc disease (DDD) with foraminal stenosis $(\mathrm{n}=96)$, spondylolisthesis $(n=75)$, recurrent herniated nucleus pulposus $(n=6)$, and

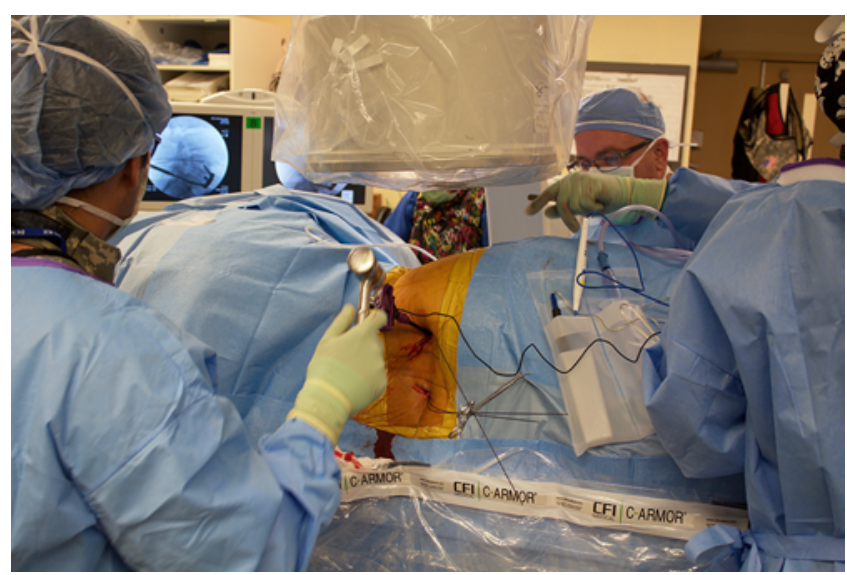

FIG. 3. With concurrent access to both the front and back of the patient, the spine surgeon can proceed with placement of PSF after L5-S1 LALIF while the assistant continues with wound closure anteriorly.

facet cyst $(n=1)$. Patient demographics are summarized in Table 1.

Bilateral PSF was placed with the patient in the lateral decubitus position in 149 patients, and 29 cases were performed stand-alone. The mean EBL was $128.3 \pm 332.8 \mathrm{ml}$. The mean case time was $101.9 \pm 41.5$ minutes: 79.3 minutes for single-level cases (51.2 minutes for stand-alone cases) and 134.5 minutes for 2-level cases. The mean length of stay was $1.64 \pm 0.9$ days. Nine patients had perioperative complications. Four patients had iliac vein injuries during LALIF that were repaired primarily: 3 without sequelae and 1 requiring delayed vascular stenting. There was 1 ureteral injury that required temporary stenting. One patient developed a retroperitoneal hematoma that required surgical evacuation. One patient had an ileus. One patient had a small cerebrovascular accident shortly after surgery without long-term sequelae. Lastly, there was 1 case of retrograde ejaculation that resolved at 6 weeks postoperatively. Operative statistics and complications are listed in Table 2.

Nine patients total required reoperation, including the evacuation of a retroperitoneal hematoma and vascular and ureteral stenting mentioned above. One patient was

\section{TABLE 1. Demographics and diagnoses in 178 patients}

\begin{tabular}{cc}
\hline & Value \\
\hline Age in yrs, mean (range) & $57(24-81)$ \\
\hline Sex, $\mathrm{n}$ & 87 \\
\hline Female & 91 \\
\hline Male & $29.55(18-46.5)$ \\
\hline BMl in kg/m², mean (range) & \\
\hline Surgical diagnosis, $\mathrm{n}(\%)$ & $96(53.9)$ \\
\hline DDD w/ foraminal stenosis & $75(42.1)$ \\
\hline Spondylolisthesis & $6(3.4)$ \\
\hline Recurrent HNP & $1(0.6)$ \\
\hline Facet cyst
\end{tabular}

$\mathrm{HNP}=$ herniated nucleus pulposus. 
TABLE 2. Operative information and complications

\begin{tabular}{lc}
\hline & No. of Patients (\%) \\
\hline Total & 178 \\
\hline LALIF at L5-S1 & 105 \\
\hline LALIF at L5-S1 + LLIF at L4-5 & 73 \\
\hline Fixation & 149 \\
\hline Bilateral PSF* & 29 \\
\hline Stand-alone & $9(5.1)$ \\
\hline Periop complications & $4(2.2)$ \\
\hline Vascular injury & $1(0.6)$ \\
\hline Ureteral injury & $1(0.6)$ \\
\hline Retroperitoneal hematoma & $1(0.6)$ \\
\hline lleus & $1(0.6)$ \\
\hline Cerebrovascular accident & $1(0.6)$ \\
\hline Retrograde ejaculation &
\end{tabular}

${ }^{*}$ In the lateral decubitus position.

found to have an incisional hernia that required repair. In another patient, the tip of a K-wire broke off during retrieval (likely due to adjustment for increased medial angulation during placement of the screw over the wire) and lodged in the lateral recess, causing new radicular pain. After laminectomy to retrieve the piece of wire, which was found nearly piercing the dura mater of the proximal nerve root, the radicular symptoms resolved. Another patient required reoperation for a malpositioned spacer that caused new radicular symptoms.

Indirect decompression failed in 3 patients (1.7\%), all of whom underwent multilevel procedures. The first patient was a 42-year-old female who underwent LSPS from L4 to $\mathrm{S} 1$ for coronal deformity and severe right L4 radicular pain. The patient's symptoms worsened after surgery, and the decision was made to perform a reoperation on postoperative day 3. During right L4 laminectomy, a large intraforaminal facet cyst was encountered and removed. The patient had complete resolution of pain thereafter. The second patient was a 70-year-old male who underwent LSPS from L4 to S1 for severe stenosis associated with spondylolisthesis at L4-5. The patient did well initially but developed recurrent pain secondary to loss of disc space height due to a migrated spacer at L4-5 (Fig. 4). The patient underwent an L4 laminectomy 3.5 months postoperatively and had resolution of pain. The third failure of indirect decompression occurred in a 48-year-old female who underwent LSPS from L1 to S1 for bilateral radiculopathy and had persistent radicular pain postoperatively. Posterior decompression was performed 6 weeks after surgery, and her leg pain eventually resolved. Patients requiring reoperation are listed in Table 3 .

\section{Discussion}

To our knowledge, this is the first study to examine the effectiveness of indirect decompression in the setting of LSPS involving LALIF at L5-S1. In this series, 3 indirect decompression failures $(1.7 \%)$ were observed, the lowest failure rate reported in studies in which there were no apparent exclusions of patients on the basis of degree or etiology of stenosis..$^{1,15-17}$ No elements of the technique, specific to the technique of ALIF in the lateral decubitus position, were identified that would otherwise allow for the relatively low rate of indirect decompression failure. ${ }^{11}$ Rather, given the complete commitment to indirect decompression in this consecutive patient series (with no exclusions of patients based on stenosis), this rate suggests a true representation of the effectiveness of indirect decompression.

The rates of additional posterior decompression after ALIF or LLIF in the literature vary widely and have been
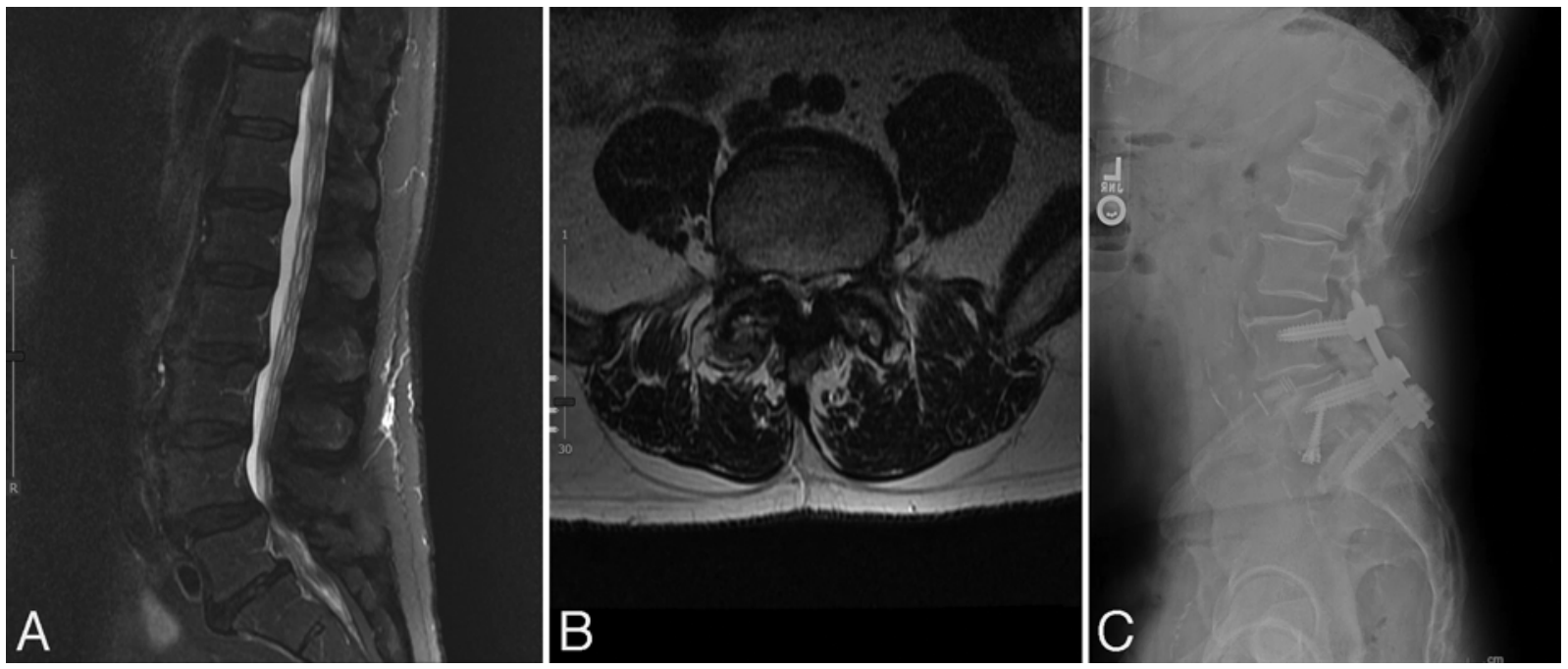

FIG. 4. Sagittal (A) and axial (B) T2-weighted MR images showing severe stenosis associated with spondylolisthesis at L4-5 and DDD at L5-S1 in a 70-year-old male with radicular pain and claudication. The patient underwent L5-S1 LALIF, L4-5 LLIF, and PSF. He did well for 1 month and then developed recurrent symptoms secondary to loss of restoration of disc height at L4-5 due to a migrated spacer (after unintentional anterior longitudinal ligament release), as seen on the lateral radiograph (C). The patient underwent an L4 laminectomy 3.5 months postoperatively and had resolution of symptoms. 
TABLE 3. Complications requiring reoperation

\begin{tabular}{lc}
\hline & No. of Complications (\%) \\
\hline Total & $9(5.1)$ \\
\hline Failure of indirect decompression & $3(1.7)$ \\
\hline lliac vein injury requiring stenting & $1(0.6)$ \\
\hline Ureteral injury requiring stenting & $1(0.6)$ \\
\hline Retroperitoneal hematoma & $1(0.6)$ \\
\hline Malpositioned spacer & $1(0.6)$ \\
\hline Hardware failure: fractured K-wire & $1(0.6)$ \\
\hline Incisional hernia & $1(0.6)$ \\
\hline
\end{tabular}

reported to be as high as $70 \% .^{18,19}$ It can be difficult to ascertain the true rate of failure of indirect decompression for 2 reasons. First, the decision to perform direct decompression is often made perioperatively. For example, Kim et al. examined clinical outcomes with a wide versus standard LLIF cage; $60 \%$ of patients underwent immediate posterior decompression. ${ }^{20}$ In another study by Park et al., of patients who underwent either LLIF or ALIF, 72\% underwent posterior decompression within the first 2.8 days after interbody fusion when preoperative radicular symptoms failed to improve. ${ }^{19}$ It is possible that in these studies the authors committed to posterior decompression prior to knowing whether it was truly necessary. While many of the patients in the current series do have persistent radicular symptoms after surgery, consistent with the literature on side effects of LLIF, ${ }^{21}$ it has been our experience that nearly all of these symptoms will resolve in 2-3 months, and thus additional surgery can be avoided.

The second reason it is difficult to know the true rate of failure of indirect decompression is that while some studies show some level of commitment to indirect decompression, they exclude some etiologies of stenosis. For example, Castellvi et al. reported outcomes on 60 patients who underwent LLIF. ${ }^{4}$ While no patients required laminectomy, patients with facet hypertrophy or a herniated disc fragment were excluded. ${ }^{4}$ In another study of indirect decompression in 28 patients undergoing LLIF by Gabel et al., only 1 patient (3.6\%) required additional posterior decompression. However, patients with free disc fragments, facet cysts, and severe stenosis were excluded. ${ }^{17}$ The notion of exclusion of patients with severe spinal stenosis is an interesting one. In the most cited paper on indirect decompression in LLIF, Oliveira et al. reported on 21 patients who underwent stand-alone LLIF. ${ }^{1}$ Two patients $(9.5 \%)$ required posterior decompression for persistent radicular symptoms. Based on these failures, the authors suggest that severe central stenosis could be a contraindication to indirect decompression. Interestingly, the 2 failures of indirect decompression occurred because of inadequate restoration of disc height, not because of the presence of severe stenosis. ${ }^{1}$ Indeed, severe central stenosis may not be a contraindication to indirect decompression. In a study by Shimizu et al., 42 patients with severe stenosis (Schizas grade C or D) underwent LLIF, and none required laminectomy. ${ }^{22}$ In addition, the average rate of expansion of CSA was $172.0 \%$ and $274.0 \%$ on MR images at 3 weeks and 1 year, respectively, suggesting that the effect of interbody fusion on severe stenosis is not only persistent but improves over time. ${ }^{22}$ While there was not a quantitative assessment of the number of patients with severe stenosis preoperatively in this study, there were also no exclusions preoperatively based on the severity of central or foraminal stenosis. Thus, we feel that the rate of $1.7 \%$ reported here may more accurately reflect the true rate of failure of indirect decompression. This low rate of failure should be weighed against the risks of performing a direct decompression.

In cases involving ALIF at L5-S1, LLIF at L4-5, and bilateral PSF, the strategy of LSPS avoids two "flips" of the patient. In a preliminary study of LLIF, Tohmeh et al. reported an average flip time from the lateral to the prone position of 38 minutes. ${ }^{23}$ This is similar to the 32-minute flip-time average at our institution (internal unpublished data). Thus, in cases involving pathology at L4-S1 that is so commonly encountered in the practice of a degenerative spine surgeon, LSPS saves over an hour of time ordinarily dedicated to repositioning the patient. While direct decompression can be performed in the lateral decubitus position, typically it requires a flip to the prone position, thereby eliminating much of the efficiency gained with LSPS. A commitment to indirect decompression would therefore maximize the efficiency benefits of LSPS.

In addition to the time to flip the patient as described above, direct decompression requires time. In a study examining the learning curve of minimally invasive laminectomy, Ahn et al. reported an average operative time of 39.4 minutes for a surgeon experienced with the procedure (the time was 52.2 minutes during the initial study period when the surgeon was relatively inexperienced). ${ }^{24}$ In a study of minimally invasive decompressions performed at an ambulatory surgery center, Patel et al. reported an operative time of 42.6 minutes. ${ }^{25}$ Even if one considers overlap of time for setup (e.g., for a decompression done in the setting of a fusion), the time needed for a flip and decompression could realistically exceed 1 hour. This extra operative time would also negate the efficiency benefits achieved with LSPS. ${ }^{26,27}$

The extra time required for direct decompression in the prone position can lead to significantly increased costs per case for lumbar fusions. ${ }^{7}$ In an era of increased vigilance on healthcare spending, these extra costs should not be discounted. More important than the financial costs, however, are the increased risks to the patient associated with the performance of the direct decompression. First, exposure of the thecal sac in laminectomy is thought to lead to epidural fibrosis and scarring, which can cause nerve root tethering and persistent compression. ${ }^{28-30}$ This resultant tethering has been implicated in persistent radiculopathy after laminectomy, and up to $24 \%$ of cases of so-called failed-back surgery syndrome..$^{28,29,31}$ In addition to the risk of epidural fibrosis, there is also the risk of inadvertent durotomy after laminectomy. In a large series of more than 1000 patients undergoing lumbar spine surgery, Takahashi et al. reported their own overall rate of durotomy of $4 \%-5 \%$ but a rate of up to $18 \%$ across the literature. ${ }^{32}$ Presumably, the more difficult decompression 

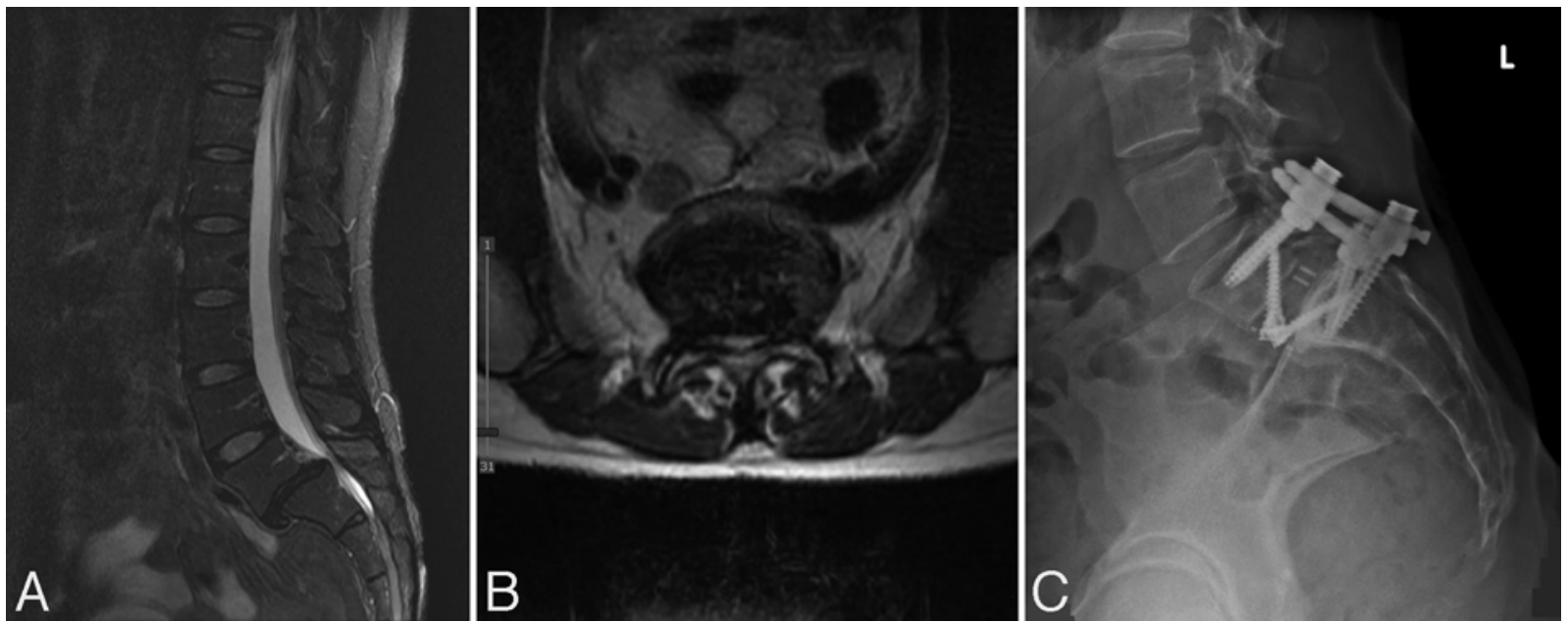

FIG. 5. Sagittal (A) and axial (B) T2-weighted MR images obtained in a 42-year-old female with severe (Schizas grade 3) stenosis secondary to near-grade II spondylolisthesis. She presented with right leg pain, numbness, and 3/5 motor weakness in plantarflexion. She was treated successfully with indirect decompression with LALIF at L5-S1 as seen on the lateral postoperative radiograph $(\mathbf{C})$.

done in a lateral decubitus position would be expected to have an even higher rate of durotomy.

In addition to the risks of direct decompression, the extra operative time needed to perform the laminectomy carries inherent risk. Several recent retrospective cohort studies of patients from the American College of Surgeons NSQIP (National Surgical Quality Improvement Program) database have shown increased operative time to be a significant risk factor for complications in lumbar spine surgery. ${ }^{33-36}$ Studies by both Kim et al. and Hersey et al. found that each independent hour of extra operative time correlated with a linear increase in the rate of several complications, including the need for transfusion, postoperative thromboembolism, urinary tract infection, and overall complication rate. ${ }^{33,34}$ In another study of elderly patients who underwent lumbar spine surgery, Saleh et al. found that patients with operative times of 120-180 minutes were nearly twice as likely to have complications than those patients with operative times under 120 minutes. ${ }^{36}$ Those with operative times greater than 180 minutes were 3 times more likely to have complications. The data from these studies clearly show that even 1 additional hour of operative time is not without serious risk of morbidity to the patient.

In this study, there was a $2.2 \%$ rate (4 patients) of vascular injury and $0.6 \%$ rate (1 patient for each) of ureteral injury, ileus, and retrograde ejaculation. The seriousness of these complications of anterior approaches to the spine should not be understated; 1 patient required delayed stenting for their vascular injury and the patient with the ureteral injury required stenting until the ureter healed. If anterior spinal reconstruction is to be undertaken in hopes of better achieving indirect decompression, surgeons should be aware of these potential complications and weigh them against the risks associated with traditional posterior approaches during which the neural elements are directly decompressed.

The limitations of this study relate primarily to the retrospective nature of the study as well as the fact that a quantitative analysis of pre- and postoperative imaging studies was not undertaken. Also, the mean follow-up interval of 10.9 months is short. Lastly, because the primary clinical outcomes of this study were binary, namely the success or failure of indirect decompression, a detailed discussion of patient-reported outcomes was not felt to be necessary. Given these limitations, the results of this study should be interpreted cautiously, pending more long-term prospective studies.

Despite our low rate of failure of indirect decompression in a relatively large series of consecutive patients, unanswered questions remain about when to rely solely on indirect decompression. First, because we did not assess the severity of central or foraminal stenosis on preoperative imaging, the findings of Shimizu et al. showing the effectiveness of indirect decompression in severely stenotic patients cannot be directly corroborated. ${ }^{22}$ Many of the current patients had severe stenosis on preoperative imaging (see Fig. 5) and were not excluded from analysis, but, unfortunately, the effectiveness of indirect decompression in this subset of patients cannot be determined from this study. Second, since only 1 patient with a facet cyst was treated successfully in this study, we cannot comment on the effectiveness of indirect decompression in the treatment of facet cysts. Third, we cannot comment specifically on the risks of failure of indirect decompression in patients with osteoporosis. It would be safe to assume that osteoporosis is a risk factor for failure, as it can lead to subsidence of the intervertebral spacer and thus failure to maintain restored disc space height. Failure to maintain restoration of disc space height was the cause of failure in Oliveira and colleagues' and other studies and was the cause of failure in the second patient in the current series. ${ }^{1,19}$ Fourth, locked facets can prevent proper restoration of disc height, and this is often cited as a potential failure of indirect decompression., ${ }^{1,43}$ Given the lack of preoperative CT evaluation (and given our small number of failures), it is not pos- 
sible to comment on whether the presence of locked facets should prompt surgeons to consider direct decompression. Fifth, we cannot comment on the effectiveness of indirect decompression in cases involving fusion at more than 2 levels. In their extensive review of indirect decompression, Lang et al. suggested that reliance on indirect decompression should be avoided in multilevel cases..$^{18}$ The fact that all 3 failures of indirect decompression occurred in multilevel cases in this study may not be coincidental. Sixth, various authors have reported that the presence of pain while recumbent leads to worse clinical outcomes with reliance on indirect decompression after LLIF. ${ }^{38,39}$ We did not stratify our patients on the basis of the presence of pain while upright versus at rest, and therefore we cannot comment on this question. Lastly, Nakashima et al. reported on 3 cases of neurological deterioration (in 158 patients) after attempted indirect decompression during LLIF for severe stenosis..$^{40}$ In all cases, the patients had severe preoperative neurological deficits that worsened after surgery. None of our patients had severe preoperative neurological deficits (other than foot weakness) or bowel or bladder dysfunction. In these cases, direct decompression of the neural elements should be considered to maximize the chances of full neurological recovery.

Because of the low number of failures of indirect decompression in this series, conclusions on risks of failure of indirect decompression cannot be made. The only constant among the 3 cases of failure was that they were all multilevel fusions. Future prospective analyses will be needed to address what effect, if any, the aforementioned factors have on the success of indirect decompression.

\section{Conclusions}

The results of this study suggest that 1) direct decompression of the neural elements after LALIF and LLIF may be unnecessary, and 2) reliance on indirect decompression allows for the maximal efficiency benefits of LSPS to be achieved. Future studies are needed to determine which specific factors lead to a higher risk of failure of indirect decompression. However, for the vast majority of 1- or 2-level degenerative cases requiring LSPS from L4 to $\mathrm{S} 1$, the rate of failure of indirect decompression appears to be very low. The very low risk of failure of indirect decompression should be weighed against the risks associated with laminectomy as well as the risks associated with the increased operative time necessary to perform the laminectomy.

\section{References}

1. Oliveira L, Marchi L, Coutinho E, Pimenta L. A radiographic assessment of the ability of the extreme lateral interbody fusion procedure to indirectly decompress the neural elements. Spine (Phila Pa 1976). 2010;35(26)(suppl):S331-S337.

2. Hsieh PC, Koski TR, O’Shaughnessy BA, et al. Anterior lumbar interbody fusion in comparison with transforaminal lumbar interbody fusion: implications for the restoration of foraminal height, local disc angle, lumbar lordosis, and sagittal balance. J Neurosurg Spine. 2007;7(4):379-386.

3. Kepler CK, Sharma AK, Huang RC, et al. Indirect foraminal decompression after lateral transpsoas interbody fusion. $J$ Neurosurg Spine. 2012;16(4):329-333.
4. Castellvi AE, Nienke TW, Marulanda GA, et al. Indirect decompression of lumbar stenosis with transpsoas interbody cages and percutaneous posterior instrumentation. Clin $\mathrm{Or}$ thop Relat Res. 2014;472(6):1784-1791.

5. Elowitz EH, Yanni DS, Chwajol M, et al. Evaluation of indirect decompression of the lumbar spinal canal following minimally invasive lateral transpsoas interbody fusion: radiographic and outcome analysis. Minim Invasive Neurosurg. 2011;54(5-6):201-206.

6. Malham GM, Wagner TP, Claydon MH. Anterior lumbar interbody fusion in a lateral decubitus position: technique and outcomes in obese patients. J Spine Surg. 2019;5(4):433-442.

7. Blizzard DJ, Thomas JA. MIS single-position lateral and oblique lateral lumbar interbody fusion and bilateral pedicle screw fixation: feasibility and perioperative results. Spine (Phila Pa 1976). 2018;43(6):440-446.

8. Yson SC, Sembrano JN, Santos ERG, et al. Does prone repositioning before posterior fixation produce greater lordosis in lateral lumbar interbody fusion (LLIF)? J Spinal Disord Tech. 2014;27(7):364-369.

9. Ziino C, Konopka JA, Ajiboye RM, et al. Single position versus lateral-then-prone positioning for lateral interbody fusion and pedicle screw fixation. J Spine Surg. 2018;4(4):717-724.

10. Ziino C, Arzeno A, Cheng I. Analysis of single-position for revision surgery using lateral interbody fusion and pedicle screw fixation: feasibility and perioperative results. J Spine Surg. 2019;5(2):201-206.

11. Hiyama A, Katoh H, Sakai D, et al. Comparison of radiological changes after single- position versus dual- position for lateral interbody fusion and pedicle screw fixation. $B M C$ Musculoskelet Disord. 2019;20(1):601.

12. Ahlquist S, Park HY, Gatto J, et al. Does approach matter? A comparative radiographic analysis of spinopelvic parameters in single-level lumbar fusion. Spine J. 2018;18(11):1999_ 2008.

13. Anderton JM. The prone position for the surgical patient: a historical review of the principles and hazards. Br J Anaesth. 1991;67(4):452-463.

14. Ozgur BM, Aryan HE, Pimenta L, Taylor WR. Extreme lateral interbody fusion (XLIF): a novel surgical technique for anterior lumbar interbody fusion. Spine J. 2006;6(4):435-443.

15. Malham GM, Parker RM, Goss B, et al. Indirect foraminal decompression is independent of metabolically active facet arthropathy in extreme lateral interbody fusion. Spine (Phila $P a$ 1976). 2014;39(22):E1303-E1310.

16. Malham GM, Parker RM, Goss B, Blecher CM. Clinical results and limitations of indirect decompression in spinal stenosis with laterally implanted interbody cages: results from a prospective cohort study. Eur Spine J. 2015;24(suppl 3):339-345.

17. Gabel BC, Hoshide R, Taylor W. An algorithm to predict success of indirect decompression using the extreme lateral lumbar interbody fusion procedure. Cureus. 2015;7(9):e317.

18. Lang G, Perrech M, Navarro-Ramirez R, et al. Potential and limitations of neural decompression in extreme lateral interbody fusion-a systematic review. World Neurosurg. 2017; 101:99-113.

19. Park D, Mummaneni PV, Mehra R, et al. Predictors of the need for laminectomy after indirect decompression via initial anterior or lateral lumbar interbody fusion. J Neurosurg Spine. 2020;32(6):781-787.

20. Kim SJ, Lee YS, Park SW, et al. Direct lateral lumbar interbody fusion: clinical and radiological outcomes. J Korean Neurosurg Soc. 2014;55(5):248-254.

21. Tohmeh AG, Rodgers WB, Peterson MD. Dynamically evoked, discrete-threshold electromyography in the extreme lateral interbody fusion approach. J Neurosurg Spine. 2011; 14(1):31-37.

22. Shimizu T, Fujibayashi S, Otsuki B, et al. Indirect decom- 
pression with lateral interbody fusion for severe degenerative lumbar spinal stenosis: minimum 1-year MRI follow-up. $J$ Neurosurg Spine. 2020;33(1):27-34.

23. Tohmeh AG, Watson B, Tohmeh M, Zielinski XJ. Allograft cellular bone matrix in extreme lateral interbody fusion: preliminary radiographic and clinical outcomes. ScientificWorldJournal. 2012;2012:263637.

24. Ahn J, Iqbal A, Manning BT, et al. Minimally invasive lumbar decompression-the surgical learning curve. Spine J. 2016;16(8):909-916.

25. Patel DV, Yoo JS, Karmarkar SS, et al. Minimally invasive lumbar decompression in an ambulatory surgery center. $J$ Spine Surg. 2019;5(suppl 2):S166-S173.

26. Shippert RD. A study of time-dependent operating room fees and how to save $\$ 100000$ by using time-saving products. Am J Cosmet Surg. 2005;22(1):25-34.

27. Abbasi H, Murphy CM. Economic performance of oblique lateral lumbar interbody fusion (OLLIF) with a focus on hospital throughput efficiency. Cureus. 2015;7(7):e292.

28. Maroon JC, Abla A, Bost J. Association between peridural scar and persistent low back pain after lumbar discectomy. Neurol Res. 1999;21(1)(suppl 1):S43-S46.

29. Ross JS, Robertson JT, Frederickson RCA, et al. Association between peridural scar and recurrent radicular pain after lumbar discectomy: magnetic resonance evaluation. Neurosurgery. 1996;38(4):855-863.

30. North RB, Campbell JN, James CS, et al. Failed back surgery syndrome: 5-year follow-up in 102 patients undergoing repeated operation. Neurosurgery. 1991;28(5):685-691.

31. Gerszten PC, Moossy JJ, Flickinger JC, et al. Inhibition of peridural fibrosis after laminectomy using low-dose external beam radiation in a dog model. Neurosurgery. 2000;46(6): 1478-1485.

32. Takahashi Y, Sato T, Hyodo H, et al. Incidental durotomy during lumbar spine surgery: risk factors and anatomic locations: clinical article. J Neurosurg Spine. 2013;18(2):165-169.

33. Kim BD, Hsu WK, De Oliveira GS Jr, et al. Operative duration as an independent risk factor for postoperative complications in single-level lumbar fusion: an analysis of 4588 surgical cases. Spine (Phila Pa 1976). 2014;39(6):510-520.

34. Hersey AE, Durand WM, Eltorai AEM, et al. Longer operative time in elderly patients undergoing posterior lumbar fusion is independently associated with increased complication rate. Global Spine J. 2019;9(2):179-184.

35. Samuel AM, Fu MC, Anandasivam NS, et al. After posterior fusions for adult spinal deformity, operative time is more predictive of perioperative morbidity, rather than surgical invasiveness: a need for speed? Spine (Phila Pa 1976). 2017; 42(24):1880-1887.
36. Saleh A, Thirukumaran C, Mesfin A, Molinari RW. Complications and readmission after lumbar spine surgery in elderly patients: an analysis of 2,320 patients. Spine J. 2017;17(8): 1106-1112.

37. Isaacs RE, Sembrano JN, Tohmeh AG. Two-year comparative outcomes of MIS lateral and MIS transforaminal interbody fusion in the treatment of degenerative spondylolisthesis: part II: radiographic findings. Spine (Phila Pa 1976). 2016;41(8) (suppl 8):S133-S144.

38. Khalsa AS, Eghbali A, Eastlack RK, et al. Resting pain level as a preoperative predictor of success with indirect decompression for lumbar spinal stenosis: a pilot study. Global Spine J. 2019;9(2):150-154.

39. Lim KZ, Daly C, Brown J, Goldschlager T. Dynamic posture-related preoperative pain as a single clinical criterion in patient selection for extreme lateral interbody fusion without direct decompression. Global Spine J. 2019;9(6):575-582.

40. Nakashima H, Kanemura T, Satake K, et al. Unplanned second-stage decompression for neurological deterioration caused by central canal stenosis after indirect lumbar decompression surgery. Asian Spine J. 2019;13(4):584-591.

\section{Disclosures}

Dr. Thomas: consultant for and royalties from NuVasive. Dr. Braly: clinical or research support for the study described from NuVasive. Dr. Menezes: consultant for and support from NuVasive for non-study-related clinical or research effort.

\section{Author Contributions}

Conception and design: Thomas, Menezes. Acquisition of data: all authors. Analysis and interpretation of data: all authors. Drafting the article: Thomas, Menezes. Critically revising the article: Thomas, Braly, Menezes. Reviewed submitted version of manuscript: Thomas, Braly, Menezes. Approved the final version of the manuscript on behalf of all authors: Thomas. Statistical analysis: Thomas.

\section{Correspondence}

J. Alex Thomas: Atlantic Neurosurgical and Spine Specialists, Wilmington, NC. thomas@atlanticneurosurgery.com. 